\title{
A Young Patient with Painfull Penile Lesions
}

\author{
Christopher Gaeta ${ }^{1,2}$, Stephen Scholand ${ }^{3}$, Brandon Blakey ${ }^{4}$, Richard Pescatore ${ }^{5}$ \\ 1. Emergency Medicine, Children's Hospital of Philadelphia, Philadelphia, USA 2. Emergency Medicine, Crozer- \\ Keystone Health System, Philadelphia, USA 3. Internal Medicine: Infectious Diseases, University of Arizona, Tuscon, \\ USA 4. Emergency Medicine, Crozer Keystone Health System, Chester, USA 5. Emergency Medicine, Crozer-Keystone \\ Health System, Chester, USA
}

Corresponding author: Christopher Gaeta, cgaeta1@swarthmore.edu

\begin{abstract}
Chancroid is a rare infection in the United States and many other developed countries. Infrequently identified as a cause of genital ulcer disease, chancroid's atypical presentation has only been reported in approximately 20 cases annually in the United States since 2011. Infection with the causative organism, Haemophilus ducreyi, leads to an erythematous papule that rapidly evolves into a pustule. Infected individuals commonly have more than one ulcer about $2 \mathrm{~cm}$ in diameter that is typically noted as painful. The base of the ulcer is usually covered with a gray or yellow purulent exudate and bleeds when scraped.
\end{abstract}

Despite a heavy focus in preclinical medical education, the notably rare chance to see and diagnose chancroid in clinical practice adds to the complicated profile of this infection's identification and subsequent treatment. Such lack of familiarity contributes to reports of accuracy of clinical diagnosis ranging from $30 \%$ to $80 \%$.

Categories: Emergency Medicine, Medical Education, Infectious Disease

Keywords: chancroid, differential, infectious diseases, medical education, case study, case report

\section{Introduction}

The following report presents a challenge shared by a vast majority of providers in the accurate diagnosis of chancroid. The rare clinical presentation of a patient with painful genital lesions with negative lab results for the typical array of sexually transmitted disease poses a challenge for a provider that ultimately requires a team-based approach that (in nearly all cases of chancroid) relies on clinical findings rather than awaiting highly specialized and costly laboratory testing to confirm the diagnosis.

Infection with the causative organism, Haemophilus ducreyi, leads to an erythematous papule that rapidly evolves into a pustule. Infected individuals commonly have more than one ulcer about $2 \mathrm{~cm}$ in diameter that is typically noted as painful. The base of the ulcer is usually covered with a gray or yellow purulent exudate and bleeds when scraped.

Received 12/09/2019

Review began 12/11/2019 Review ended 12/14/2019 Published 12/16/2019

\section{() Copyright 2019}

Gaeta et al. This is an open access article distributed under the terms of the Creative Commons Attribution License CC-BY 3.0., which permits unrestricted use, distribution, and reproduction in any medium, provided the original author and source are credited.
Despite a heavy focus in preclinical medical education, the notably rare chance to see and diagnose chancroid in clinical practice adds to the complicated profile of this infection's identification and subsequent treatment. Such lack of familiarity contributes to reports of the accuracy of clinical diagnosis ranging from $30 \%$ to $80 \%$.

The difficulties of accurately diagnosing the infection begin with the rarity of its presentation. More specifically, roughly about 20 cases on an annual basis have been reported by providers in the United States in the past two decades. The added barriers to obtaining a confirmed diagnosis are unrealistic to many providers. This is directly due to the absence of rapid and widespread availability for testing of chancroid. The highly specialized nature of a definitive test for chancroid is a means of diagnosis not within a realistic means of access for an overwhelming majority of providers [1-2]. As such, Center for Disease Control and Prevention (CDC) guidelines and clinical practice provide for presumptive diagnosis and treatment in the correct clinical setting [3-4].

\section{Case Presentation}

A 29-year-old male presented to the ED with painful penile lesions that had progressed for approximately five weeks prior to presentation. He had recently been released from a correctional facility. The patient reported unprotected sex with multiple male and female partners. The patient stated that the lesions began as small erythematous macules that slowly progressed to ulcers. Urine polymerase chain reaction (PCR) for gonorrhea and chlamydia, fourth generation HIV testing, rapid plasma regain (RPR) screening for syphilis, and herpes simplex virus (HSV) DNA testing of the lesions were all negative. The ulcers were cultured; however, special media for $\mathrm{H}$. ducreyi were not available at our institution and standard culture techniques grew only Staphylococcus epidermidis [5] (Figure 1). 


\section{Cureus}

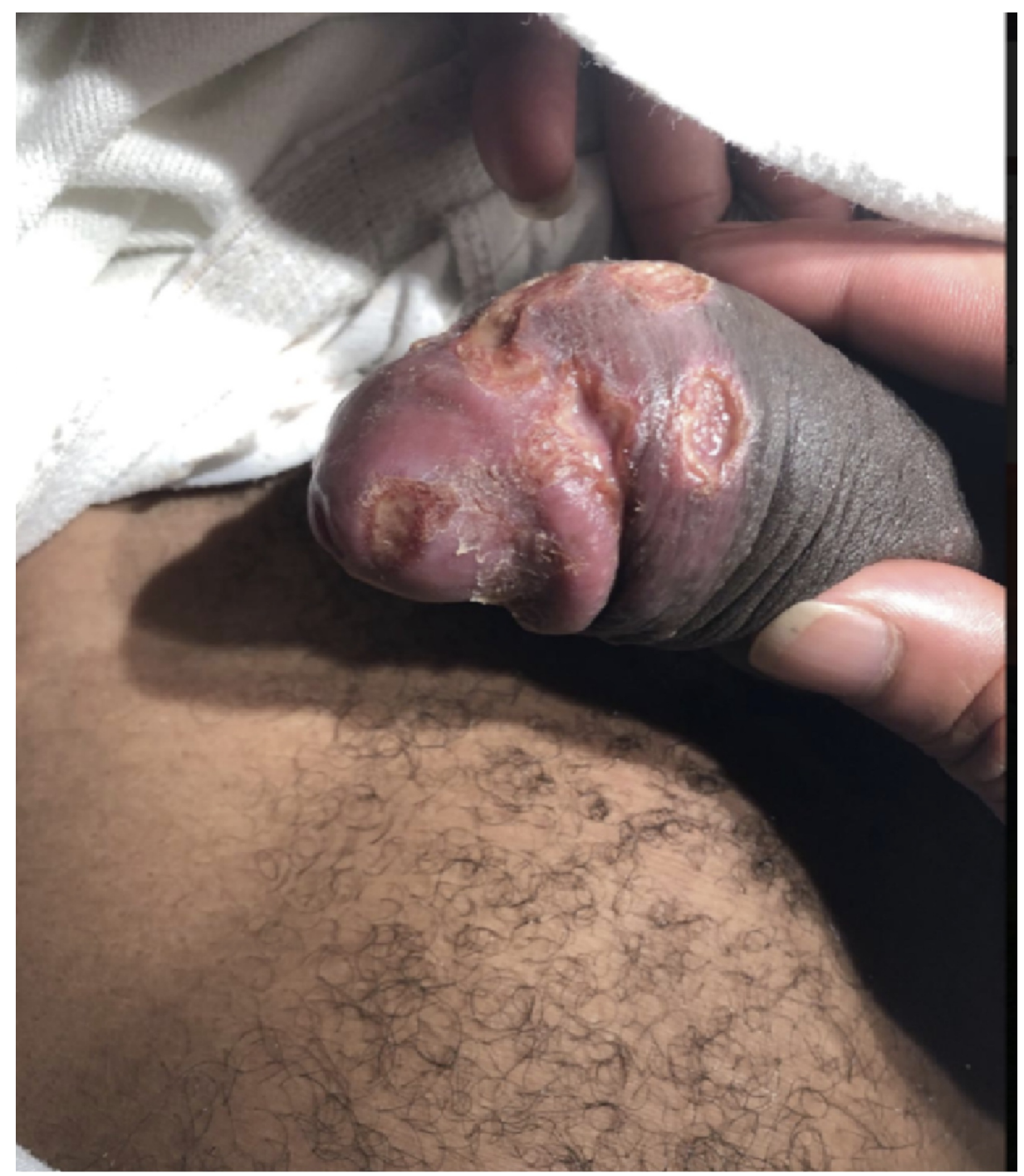

FIGURE 1: Lesions on the glans and distal penile shaft.

\section{Discussion}

The patient was treated for a diagnosis of probable chancroid with $250 \mathrm{mg}$ IM ceftriaxone and $1000 \mathrm{mg}$ oral azithromycin. At three-week follow-up, the lesions had nearly resolved compared to the initial presentation several weeks prior. This resolution is consistent with the expected course, as in cases of chancroid, clinical improvement usually occurs promptly after treatment is initiated.

Relief of pain is noted by most patients within two to three days, and objective improvement in ulcers is usually apparent within a week. Both of these were consistent with the patient's progress after his first three weeks of treatment initiation. Patients with chancroid should abstain from sex until ulcers have dried/resolved, and condom use should be stressed [6].

Providers should be aware of the presentation of chancroid in individuals who are confirmed to not have more typical sexually transmitted diseases. Scarce diagnostic media to diagnose chancroid in a laboratory should be considered as noted in the introduction. This added challenge to a provider suspecting chancroid as a diagnosis is augmented by limited educational opportunities to expose a case to providers in training. Therefore, as followed in this particular case report, clinicians should particularly begin to consider chancroid in patients with genital ulcers after more common sexually transmitted diseases such as HSV or syphilis is investigated based on the case presentation [7-8].

Underscoring the educational significance of this case, CDC data dating back to 1941 reinforces the rare presentation of chancroid on a national scale (Figure 2). 


\section{Cureus}

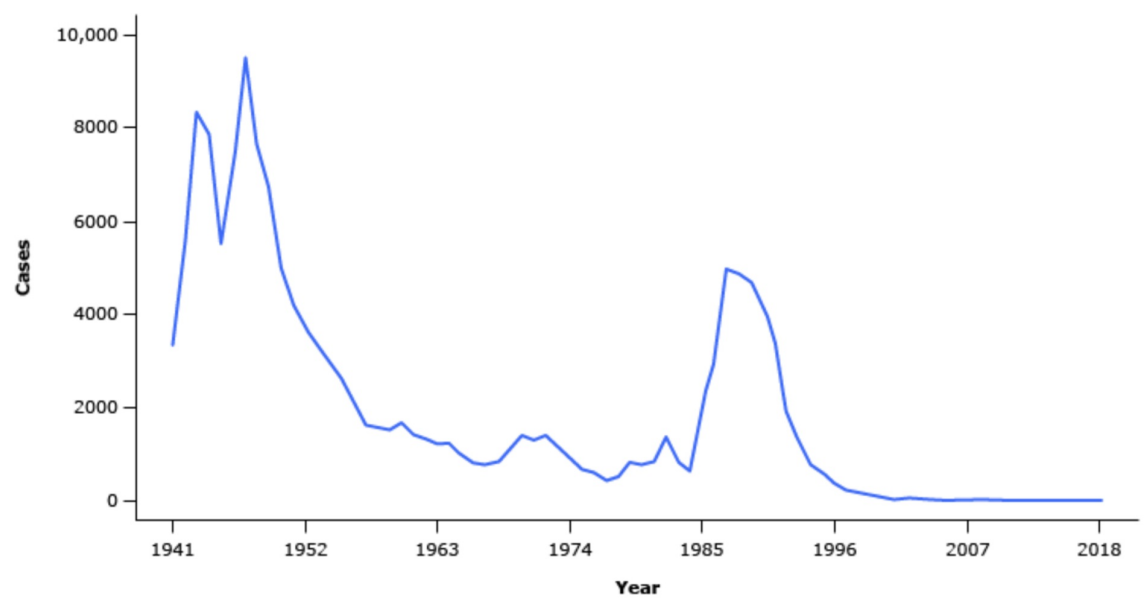

Reproduced from: Centers for Disease Control and Prevention. Sexually Transmitted Diseases Surveillance 2018. Available at: https://www.cdc.gov/std/stats18/default.htm (Accessed on October 17, 2019).

FIGURE 2: CDC report, prevalence of chancroid in the United States in the years, 1941-2018.

CDC, Center for Disease Control and Prevention

In past decades when chancroid has appeared, transmission has been typically moderate to large and localized [4]. As such, it is critical for clinicians to recognize this relatively rare clinical entity and administer presumptive treatment, especially as confirmatory testing is unlikely to be obtainable.

\section{Conclusions}

This case highlights the challenges that providers face in recognizing and diagnosing chancroid. Perhaps the most significant challenge is accurately diagnosing chancroid rests with the inability to obtain confirmatory microbiological testing. Laboratories with the ability to test for $H$. ducreyi via special culture media are not only highly specialized and costly but also scarce in the United States and abroad. This obstacle has made it difficult to estimate the true incidence rate. As such, providers rely on clinical criteria and differential diagnosis to confirm the presentation of chancroid.

\section{Additional Information}

\section{Disclosures}

Human subjects: Consent was obtained by all participants in this study. Conflicts of interest: In compliance with the ICMJE uniform disclosure form, all authors declare the following: Payment/services info: All authors have declared that no financial support was received from any organization for the submitted work. Financial relationships: All authors have declared that they have no financial relationships at present or within the previous three years with any organizations that might have an interest in the submitted work. Other relationships: All authors have declared that there are no other relationships or activities that could appear to have influenced the submitted work.

\section{References}

1. Alfa M: The laboratory diagnosis of Haemophilus ducreyi . Can J Infect Dis Med Microbiol. 2005, 16:31-34. $10.1155 / 2005 / 851610$

2. Sexually Transmitted Disease Surveillance 2018: United States Centers for Disease Control and Prevention . (2018). Accessed: 11-10-2019: https://www.cdc.gov/std/stats18/STDSurveillance2018-full-report.pdf.

3. O'Farrell N, Lazaro N: UK National Guideline for the management of chancroid. Int J STD AIDS . 2014, 25:975-983.

4. DiCarlo RP, Martin DH: The clinical diagnosis of genital ulcer disease in men . Clin Infect Dis. 1997, 25:292298. 10.1086/514548

5. Mertz KJ, Trees D, Levine WC, et al.: Etiology of genital ulcers and prevalence of human immunodeficiency virus coinfection in 10 US cities. The Genital Ulcer Disease Surveillance Group. J Infect Dis. 1998, 178 :17951798.

6. Crosby RA, Charnigo RA, Weathers C, et al.: Condom effectiveness against non-viral sexually transmitted infections: a prospective study using electronic daily diaries. Sex Transm Infect. 2012, 88:484-489. 10.1136/sextrans-2012-050618

7. Lewis DA, Mitjà O: Haemophilus ducreyi: from sexually transmitted infection to skin ulcer pathogen . Curr Opin Infect Dis. 2016, 29:1. 


\section{Cureus}

8. Gomes Naveca F, Sabidó M, Amaral Pires de Almeida T, et al.: Etiology of genital ulcer disease in a sexually transmitted infection reference center in Manaus, Brazilian Amazon. PLoS One. 2013, 8:e63953. 